\section{WEB WATCH}

- (http://vaccines.org/)

\section{Vaccines on the Web}

Vaccines and vaccine safety are hot topics in the news these days (see the article on $\mathrm{p} 160$ of this issue, by Wilson and Marcuse). But where do you go to find up-to-date vaccine news and web resources?

The Vaccine Page is a

regularly updated website that is partly supported by the Bill and Melinda Gates Children's Vaccine Program. By clicking through to the news site, readers can access timely articles from various international news sources. But The Vaccine Page is more than just a news service. The site also provides an annotated database of vaccine resources on the web, assembled and checked by the editors of UniScience News Net. Vaccine resources are listed by country and by category, for ease of navigation. Category sections are handily organized into those directed at parents, researchers and medical practitioners, and there are also sections focusing on journal access and vaccine organizations.

The Vaccine Page is a member of the Allied Vaccine Group (AVG), which consists of websites "dedicated to presenting valid scientific information about the sometimes confusing subject of vaccines" and which clearly promotes the benefits of vaccination. AVG members believe that the benefits of immunization far outweigh the risks. The publishers of The Vaccine Page also maintain the website of the AVG, and The Vaccine Page uses the AVG search engine VACSearch PLUS, which returns results from the websites of all members of the AVG.

So, if you want to access vaccine news and links to web resources which are pro-vaccination, check out The Vaccine Page today.

Elaine Bell

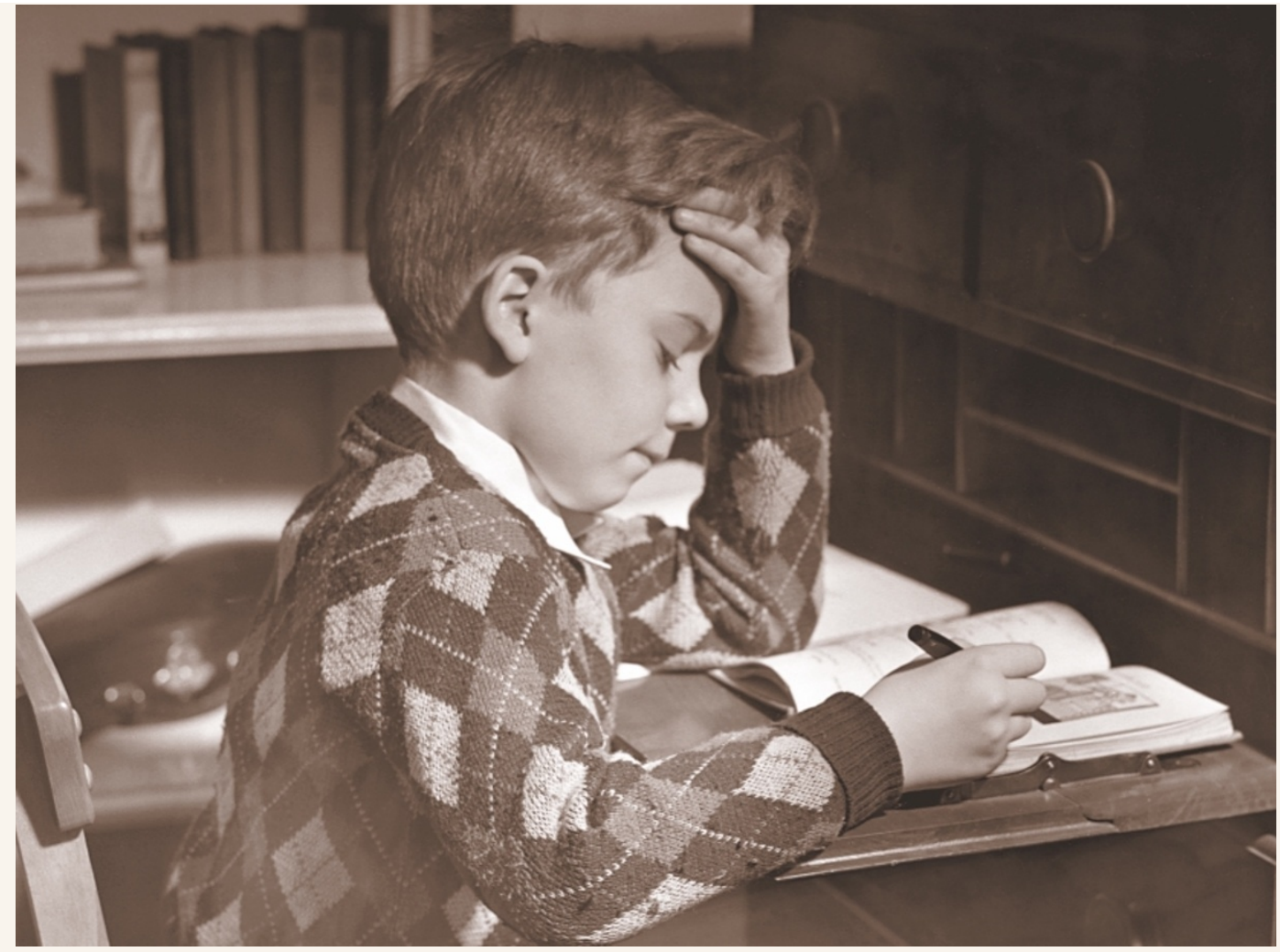

T-CELL MEMORY

\title{
Learning to remember
}

Memory is a complex business - no less so in the immune system than in the nervous system. Typically, exposure to antigen during infection induces an immune response in which effector $\mathrm{T}$ cells develop that fight the infection. Memory T cells also develop, conferring on the host the ability to mount a rapid and augmented response on subsequent exposure. The development of immunological memory is not yet fully understood, but many immunologists subscribe to the view that memory $\mathrm{T}$ cells are quiescent cells that develop from fully differentiated effector $\mathrm{T}$ cells. Now, two papers from von Andrian's group describe the cytokine-stimulated development of memory $\mathrm{T}$ cells in the absence of prior effector T-cell differentiation, and the migratory properties of these cells.

Reporting in the Journal of Clinical Investigation, Manjunath and colleagues made use of T-GFP mice, in which naive and early-activated T cells uniformly express green fluorescent protein, but terminally differentiated cytotoxic T lymphocytes (CTLs) lose this expression. T-GFP mice were crossed with transgenic P14 mice, which express a T-cell receptor specific for the lymphocytic choriomeningitis virus glycoprotein peptide (gp33-41) epitope. Naive T cells from these doubly transgenic mice were stimulated with gp33-41 peptide for 2 days, then washed and cultured with either interleukin (IL)-2 or IL-15 for a further 5 days, and their phenotype analysed. T cells exposed to high doses of IL-2 developed phenotypic and functional characteristics of effector cells - the cells were large, they had lost GFP expression, they did not express CCR7 (a chemokine receptor whose expression is retained on a subset of memory $\mathrm{T}$ cells termed central memory cells), they produced interferon $-\gamma$ and exhibited peptide-specific cytotoxicity. By contrast, naive cells exposed to IL-15 acquired various features associated with memory T cells - the cells were small, they retained GFP and CCR7 expression, they lost expression of various activation markers, and were not cytotoxic. The memory-like cells were assessed for their ability to respond to secondary antigen exposure in adoptivetransfer experiments - the exposed $\mathrm{T}$ cells survived for several weeks after transfer and were able to respond to subsequent antigen challenge.

The second paper, by Weninger and colleagues published in the Journal of Experimental Medicine, examined the migratory properties of these cells. IL-2stimulated effector $T$ cells preferentially accumulated in inflammed tissues and were excluded from most lymphoid organs. Central memory-like, IL-15exposed cells homed to lymphoid organs, such as lymph nodes and Peyer's patches, were only moderately capable of homing to inflammed tissues, and rapidly responded to recall antigen.

So, these results indicate that differentiation of CD8 $\mathrm{T}$ cells into effector cells is not necessary for the development of memory $T$ cells, and support the concept proposed by Lanzavecchia's group of the existence of distinct subsets of memory cells.

Elaine Bell

(4) References and links

ORIGINAL RESEARCH PAPERS Manjunath, N. et al. Effector differentiation is not prerequisite for generation of cytotoxic T lymphocytes. J. Clin. Invest. 108, 871-878 (2001) | Weninger, W., Crowley, M. A., Manjunath, N. \& von Andrian, U. H. Migratory properties of naive, effector, and memory CD8+ T cells. J. Exp. Med. 194, 953-966 (2001) FURTHER READING Sallusto, F., Mackay, C. R. \& Lanzavecchia, A. The role of chemokine receptors in primary, effector, and memory immune responses. Annu. Rev. Immunol. 18, 593-620 (2000) WEB SITE

Ulrich von Andrian's lab: http://cbrweb.med.harvard.edu/ uva/ 